PRECISIÓNDIAGNÓSTICAY

LIMTAACIONES DE LOS TESTNO

INVASIVOS EN PACIENTES CON

ESTEATOSIS HEPATICA METABOLICA

\title{
DIAGNOSTIC ACCURACY AND LIMITATIONS OF NON-INVASIVE TESTS IN PATIENTS WITH METABOLIC- ASSOCIATED FATTY LIVER DISEASE
}

\section{Sánchez Torrijos Y', Lucena Valera A', Ampuero Herrojo J',2,3}

'Hospital Universitario Virgen del Rocío. Sevilla.

${ }^{2}$ Instituto de Biomedicina de Sevilla. Sevilla.

${ }^{3}$ Universidad de Sevilla. Sevilla.

\section{Resumen}

La incidencia de la enfermedad del hígado graso asociada al metabolismo (MAFLD) ha aumentado en los últimos años debido al estilo de vida actual. La biopsia de hígado sigue siendo la herramienta estándar de oro para detectar y estadificar MAFLD. Por otro lado, se están desarrollando múltiples biomarcadores y pruebas no invasivas para superar las limitaciones de la biopsia hepática, incluidos el costo y la invasividad. Las pruebas no invasivas se centran principalmente en la esteatosis y, en particular, en la fibrosis hepática y se pueden clasificar en pruebas patentadas y no patentadas. Si bien hepatic steatosis index y fatty liver index son las pruebas más comunes utilizadas para la detección de esteatosis, Hepamet Fibrosis Score, NAFLD fibrosis score, FIB-4, OWLiver ${ }^{\circledR}$, y ELF ${ }^{\circledR}$ son las más utilizadas para la fibrosis hepática. Sin embargo, las pruebas no invasivas también tienen limitaciones que conviene resaltar ya que sus resultados podrían verse afectados por la presencia de diabetes, obesidad o por edades extremas que podrían dar lugar a falsos positivos o negativos. Para maximizar la precisión de los tests no invasivos, se han propuesto diferentes combinaciones integradas en algoritmos escalonados. Esta revisión tiene como objetivo destacar las fortalezas y limitaciones de dichos tests para detectar y estadificar MAFLD.

Palabras clave: esteatosis hepática metabólica, tests no invasivos.

\section{Abstract}

The incidence of metabolic-associated fatty liver disease (MAFLD) has increased in recent years due to the current lifestyle. The liver biopsy remains as the gold standard tool to detect and stage MAFLD. On the other hand, multiple biomarkers and non-invasive tests are being developed to overcome the pitfalls of liver biopsy, including cost and invasiveness. The non-invasive tests mainly focus on steatosis and, particularly, on liver fibrosis and can be classified in patented and non-patented tests. While hepatic steatosis index and fatty liver index are the most common tests for steatosis, Hepamet Fibrosis Score, NAFLD fibrosis score, FIB-4, OWLiver ${ }^{\circledR}$, and ELF ${ }^{\circledR}$ are positioned for liver fibrosis. However, non-invasive tests also have limitations that should be highlighted since their results could be affected by the presence of diabetes, obesity, or by extreme ages that could lead to false-positive or negative results. In order to maximize the accuracy 
of the non-invasive tests, different combinations integrated into stepwise algorithms have been proposed. This review aims to remark the strengths and limitations of non-invasive tests for detecting and staging MAFLD.

Keywords: MAFLD, NITS.

\section{Introduction}

Metabolic-associated fatty liver disease (MAFLD) is a clinical condition characterized by the deposition of fat in the liver, accompanied by an inflammatory component (non-alcoholic steatohepatitis (NASH)) and, finally, hepatic fibrosis ${ }^{1,2}$ in the absence of other etiologies. Currently, probably influenced by the current lifestyle, the prevalence of MAFLD is about $25-30 \%$ in the general population ${ }^{3,4}$ and more elevated in patients showing metabolic features, such as obesity and diabetes mellitus. In fact, the European Association for the Study of the Liver ${ }^{5}$ recommends screening for MAFLD in the presence of insulin resistance or metabolic syndrome. Although a small proportion of patients suffers from an advanced MAFLD, due to its high prevalence, it represents the first cause of liver disease in the Western world ${ }^{6}$ and the second indication for liver transplantation in the USA ${ }^{7}$.

The liver biopsy is the gold standard to detect and stage MAFLD. However, given the significant number of at-risk patients and the inherent limitations of the biopsy (invasiveness, poor acceptability, sampling variability, and cost), there is an unmet need to find noninvasive and cost-effective tests for the diagnosis and staging of MAFLD. Non-invasive methods should aim to diagnose patients with MAFLD and, on the other hand, identify those with a worse prognosis and/or at-risk of disease progression. They should also be helpful for the subsequent monitoring and assessment of the response to the treatment. Of note, although the levels of alanine aminotransferase (ALT) and aspartate aminotransferase (AST) may be elevated up to four times in MAFLD patients ${ }^{8}$, they can be also normal or minimally elevated and do not correlate with the fibrosis stage ${ }^{9}$. Therefore, their monitoring is not recommended for MAFLD management.

This review aims to revise the different predictive models and non-invasive tests (NITS) used to detect fibrosis in the MAFLD scenario, including their limitations in clinical practice.

\section{The spectrum of MAFLD and the use of non-invasive tests}

The diagnosis of MAFLD is based on detecting steatosis defined as fat in $\geq 5 \%$ of hepatocytes by histology ${ }^{10}$, in the absence of a relevant alcohol intake. There are currently several scores that assess hepatic steatosis based on epidemiological, biochemical, and anthropometric data. For example, fatty liver index (FLI), hepatic steatosis index $(\mathrm{HSI})$, and SteatoTest ${ }^{\circledR}$ have been validated externally in the general population showing an optimal accuracy for detecting steatosis (AUROC $>0.80)^{11,12}$. The main advantages of these scores are their wide availability, their easy implementation and interpretation, and the low cost. However, its usefulness is limited due to the low prognostic implication.

On the other hand, NASH is characterized by the presence of steatosis, hepatocellular damage (e.g., ballooning, apoptosis, necrosis), and lobular inflammatory infiltration ${ }^{13}$. The diagnosis of NASH provides important prognostic information and indicates an increased risk of progression to fibrosis, cirrhosis, and extrahepatic events $^{13}$. Non-invasive diagnosis of NASH represents a current challenge, opposite to steatosis and fibrosis, since there is a lack of non-patented NITs showing optimal results. NASHTest ${ }^{\circledR}$ is an algorithm consisting of a combination of age, sex, height, weight, and serum levels of triglycerides, cholesterol, alpha 2 macroglobulin, apolipoprotein Al, haptoglobin, GGT, ALT, AST, and total bilirubin. The AUROC of NASHTest ${ }^{\circledR}$ for the diagnosis of NASH was 0.79 (95\%C I 0.69-0.86) for the estimation and $0.79(95 \% \mathrm{Cl} 0.67-0.87)$ for the validation cohort ${ }^{14}$. One of the more novel techniques used to identify NASH markers is to detect molecules highly related to NASH using genomic, epigenomic, metabolomic, transcriptomic, or proteomic (-omic approaches) ${ }^{16}$. OWLiver ${ }^{\circledR}$ is a laboratory test in which different serum lipids and amino acids are measured and combined to define a triglyceride panel that helps differentiate between a normal liver and MAFLD, including distinguishing NASH from a fatty liver. OWLiver ${ }^{\circledR}$ showed an AUROC, sensitivity, and specificity of $0.88,0.94$, and 0.57 , respectively, for the discrimination between MAFLD and normal liver, and $0.79,0.70$, and 0.81 , respectively, for the discrimination between NASH and simple steatosis ${ }^{15}$. Figure 1 summarizes the spectrum of NAFLD and the main NITs stage by stage.

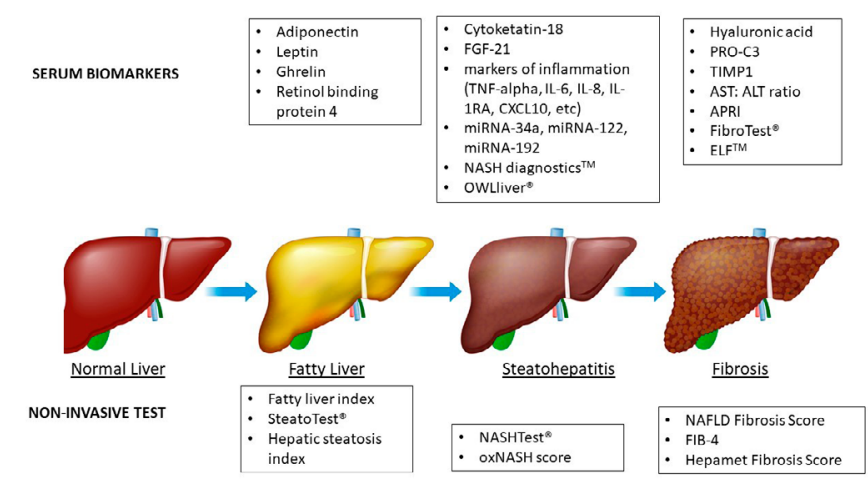

Figure 1

Serum biomarkers and non-invasive tests in MAFLD. FGF-21, fibroblast growth factor 21; TIMP1, tissue inhibitor of metalloproteinases 1; CXCL10, chemokine 10; IL-1RA, IL-1 receptor antagonist protein; miRNAs, microRNAs; PRO-C3, a marker of type III collagen formation; APRI, AST: platelet ratio index; ELF, enhanced liver fibrosis.

Finally, fibrosis is the progressive deposition of extracellular matrix in the liver parenchyma that precedes the development of cirrhosis, representing the most important prognostic factor in MAFLD ${ }^{17}$. Therefore, despite having NITs for the different components of the disease, those detecting fibrosis are more relevant in clinical practice. As mentioned above, although biopsy is the gold standard for diagnosing MAFLD and fibrosis, there is currently a greater acceptance of NITs as an alternative to biopsy, and these are being incorporated into therapeutic and diagnostic algorithms.

The combination of several parameters is considered adequate for the diagnosis of fibrosis in MAFLD. Among non-patented tests, NAFLD Fibrosis Score (NFS), FIB-4, and Hepamet Fibrosis Score (HFS) are the most commonly used ${ }^{19}$. Both NFS and FIB-4 have been validated externally in MAFLD populations, showing optimal results (AUROC around 0.80$)^{20}$. For FIB-4, thresholds of 2.67 and 3.25 showed sensitivities of $26.6 \%$ and $31.8 \%$, and specificities of $96.5 \%$ and $96.0 \%$, respectively. For NFS, a threshold of 1.455 had a sensitivity and a 
specificity of $72 \%$ and $70 \%$, respectively. FS, which includes albumin, platelets, AST, HOMA, age, and sex, has been recently published, showing a significantly higher diagnostic accuracy than NFS and FIB-

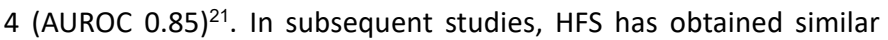
AUROCs $(0.94(0.90-0.99,95 \% \mathrm{Cl})$ for predicting advanced fibrosis in patients with chronic viral liver disease and MAFLD22. On the other hand, AST:ALT ratio and AST: platelet ratio index (APRI) are simple NITs for detecting advanced fibrosis on MAFLD ${ }^{10}$, although they were initially derived from chronic hepatitis $C$ cohorts $^{23}$. Patented or composite biomarkers have demonstrated an improvement in diagnostic precision compared to non-patented scores; however, their application remains limited due to their cost and availability ${ }^{18}$. For this reason, patented biomarkers are usually reserved for patients with undetermined results for advanced fibrosis after using nonpatented NITs ${ }^{16}$. FibroTest $^{\circledast}$ is a commercially available panel that uses seven parameters, including alpha 2 macroglobulin, haptoglobin, and apolipoprotein-A1, and showed a high AUROC $(0.88)$ to predict advanced fibrosis in MAFLD ${ }^{24}$. On the other hand, the enhanced liver fibrosis (ELFTM), which also measures three molecules involved in liver matrix metabolism ${ }^{25}$, has been used to improve the detection of advanced fibrosis in those with indeterminate fibrosis by other methods ${ }^{26}$.

\section{Limitations of non-invasive tests in MAFLD}

Despite many serum biomarkers and combination panels are published in the literature, there is a lack of independent validation, uncertainty regarding optimum diagnostic cut-off, and a lack of knowledge regarding the influence of other factors that might affect the test interpretation. For instance, although FIB-4, HFS, and NFS have shown promising results, they have some limitations that must be considered (Table 1). In addition, sometimes having lowprevalence populations precludes calculating some operating features of the NITs for advanced fibrosis since, in most studies, the patients who classify initially as low- or high-risk are not biopsied ${ }^{27}$.

\section{Influence of age}

Non-invasive indexes of fibrosis have been developed and validated mainly in patients between 35 and 65 years of age. In patients over 65 years, a high rate of false positives has been observed for advanced fibrosis, drastically decreasing the NFS and
FIB-4 specificity in these patients. McPherson et al. ${ }^{28}$ established ageadjusted cut-off values to improve the specificity in these patients. By adopting the revised threshold in patients older than 65 years of age (FIB-4> 2.0 and NFS> 0.12 for the lower cut-offs), the sensitivity and specificity achieved for advanced fibrosis were $77 \%$ and $70 \%$; and $80 \%$ and $70 \%$, respectively, compared to the previous cut-off values (specificity $20 \%$ for NFS $>-1.445$ and $35 \%$ for FIB-4> 1.3). Likewise, non-invasive liver fibrosis scores in adults $<35$ years and, mainly, in pediatric patients have a low capacity to diagnose significant fibrosis with AUROCs between 0.36-0.61 29,30 .

\section{Influence of diabetes mellitus}

The diagnostic precision of NITs for advanced fibrosis in patients with MALFD and diabetes mellitus is lower than in patients without it. Bertot et al. ${ }^{32}$ demonstrated a lower AUROC for FIB-4, NFS, and APRI in patients with DM. Also, they observed that up to $21 \%$ and $27 \%$ of patients with diabetes mellitus, despite showing a low risk according to NITs, suffered ultimately from decompensation and hepatocarcinoma, respectively.

\section{Influence of obesity}

Up to $90 \%$ of obese patients have MAFLD. Considering this, NITs have not been correctly validated in the obese population despite they have a high risk of fibrosis. In fact, lower diagnostic precision has been observed for FIB-4, APRI, and NFS in obese patients, especially those methods that include BMI in their algorithm ${ }^{33}$. This parameter does not significantly discriminate the risk of fibrosis and may, therefore, bias the results in obese patients. Joo et al. ${ }^{34}$ demonstrated significantly higher FIB-4 and NFS AUROCs in non-obese patients compared with the obese subgroup (FIB-4: 0.965 vs. 0.835 , and NFS: 0.965 vs. 0.801$)$.

\section{Influence of liver damage}

NITs identifying steatosis (FLI, HSI, and SteatoTest ${ }^{\circledR}$ ) have been externally validated. However, they are influenced by fibrosis and inflammation. Consequently, they are not able to accurately quantify steatosis in situations of great liver inflammation or advanced fibrosis $^{31}$.

\begin{tabular}{|c|c|c|c|c|}
\hline \multicolumn{5}{|c|}{ Table 1. Pros and cons of using NITs to detect advanced fibrosis in MAFLD. } \\
\hline & & NFS & FIB 4 & HFS \\
\hline AUROC & & $0.78-0.82$ & $0.78-0.84$ & $0.85-0.94$ \\
\hline Indeterminate area & & $31 \%$ & $26 \%$ & $21 \%$ \\
\hline Advantages & & $\begin{array}{l}\text { External validation } \\
\text { Heterogenous and extensive } \\
\text { cohort } \\
\text { Low cost }\end{array}$ & $\begin{array}{l}\text { External validation } \\
\text { Heterogenous and extensive } \\
\text { cohort } \\
\text { Low cost }\end{array}$ & $\begin{array}{l}\text { External validation } \\
\text { Heterogenous and extensive } \\
\text { cohort } \\
\text { Low cost }\end{array}$ \\
\hline \multirow[t]{3}{*}{ Disadvantages } & $\begin{array}{l}\text { Age }<35 \\
\text { or }>65\end{array}$ & Moderate-to-high influence & Moderate-to-high influence & Low influence \\
\hline & Obesity & High influence & Moderate-to-high influence & Low-to-moderate influence \\
\hline & Diabetes & Moderate-to-high influence & Moderate-to-high influence & Low-to-moderate influence \\
\hline
\end{tabular}




\section{Undetermined results from NITs}

One of the main concerns of NITs is the high percentage of patients located in the grey zone (undetermined results) for advanced fibrosis, representing up to $25-30 \%$ of patients for NFS and FIB-4 $4^{35,36}$. By contrast, HFS, although it maintains a grey zone, is able to reduce significantly the number of patients allocated at risk of indeterminate fibrosis ${ }^{37}$. In this setting, an alternative for patients with initial results of indeterminate fibrosis is to use more than one NIT to increase the accuracy of the detection of mild or advanced fibrosis ${ }^{27}$.

\section{Conclusion}

There are a multitude of serum biomarkers and NITs currently accessible for MAFLD. Their usefulness for detecting steatosis and fibrosis is optimal, although the diagnosis of steatohepatitis remains a challenge. The balance between patented and non-patented, as well as the combination of NITs, should represent an interesting approach to overcome the limitations that they show. Therefore, biomarkers and NITs should represent the first step in the diagnosis of MAFLD.

\section{Bibliografía}

1. Ma X, Li Z. Pathogenesis of non-alcoholic steatohepatitis (NASH). Chin J Dig Dis. $2006 ; 7(1): 7-11$.

2. Eslam M, Sanyal AJ GJICP. MAFLD: A Consensus-Driven Proposed Nomenclature for Metabolic Associated Fatty Liver Disease. Gastroenterology. 2020;158(7):1999-2014.

3. Barrera F, George J. The Role of Diet and Nutritional Intervention for the Management of Patients with NAFLD. Clin Liver Dis. 2014;18(1):91-112.

4. Gerber L, Otgonsuren M, Mishra A, Escheik C, Birerdinc A, Stepanova M, et al. Non-alcoholic fatty liver disease (NAFLD) is associated with low level of physical activity: a population-based study. Aliment Pharmacol Ther. 2012;36(8):772-81.

5. Byrne CD, Targher G. EASL-EASD-EASO Clinical Practice Guidelines for the management of non-alcoholic fatty liver disease. Diabetologia. 2016;59(6):1141-4.

6. McPherson S, Hardy T, Henderson E, Burt AD, Day CP, Anstee QM. Evidence of NAFLD progression from steatosis to fibrosing-steatohepatitis using paired biopsies: implications for prognosis and clinical management. J Hepatol. 2015;62(5):1148-55.

7. Wong RJ, Aguilar M, Cheung R, Perumpail RB, Harrison SA, Younossi ZM, et al. Nonalcoholic Steatohepatitis Is the Second Leading Etiology of Liver Disease Among Adults Awaiting Liver Transplantation in the United States. Gastroenterology. 2015;148(3):547-55.

8. Mofrad P, Contos MJ, Haque M, Sargeant C, Fisher RA, Luketic VA, et al. Clinical and histologic spectrum of non-alcoholic fatty liver disease associated with normal ALT values. Hepatology. 2003;37(6):1286-92.
9. Fracanzani AL, Valenti L, Bugianesi E, Andreoletti M, Colli A, Vanni E, et al. Risk of severe liver disease in non-alcoholic fatty liver disease with normal aminotransferase levels: a role for insulin resistance and diabetes. Hepatology. 2008;48(3):792-8.

10. Wong VW-S, Adams LA, de Lédinghen V, Wong GL-H, Sookoian S. Noninvasive biomarkers in NAFLD and NASH- current progress and future promise. Nat Rev Gastroenterol Hepatol. 2018;15(8):461-78.

11. Lee J-H, Kim D, Kim HJ, Lee C-H, Yang JI, Kim W, et al. Hepatic steatosis index: a simple screening tool reflecting non-alcoholic fatty liver disease. Dig Liver Dis. 2010;42(7):503-8.

12. Calori G, Lattuada G, Ragogna F, Garancini MP, Crosignani P, Villa M, et al. Fatty liver index and mortality: The cremona study in the 15th year of followup. Hepatology. 2011;54(1):145-52.

13. Wong VW-S, Chan W-K, Chitturi S, Chawla Y, Dan YY, Duseja A, et al. AsiaPacific Working Party on Non-alcoholic Fatty Liver Disease guidelines 2017 Part 1: Definition, risk factors and assessment. J Gastroenterol Hepatol. 2018;33(1):70-85.

14. Poynard T, Ratziu V, Charlotte F, Messous D, Munteanu M, Imbert-Bismut F, et al. Diagnostic value of biochemical markers (NashTest) for the prediction of non alcoholo steato hepatitis in patients with non-alcoholic fatty liver disease. BMC Gastroenterol. 2006. 10;6(1):34

15. Mayo R, Crespo J, Martínez-Arranz I, Banales JM, Arias M, Mincholé I, et al. Metabolomic-based noninvasive serum test to diagnose nonalcoholic steatohepatitis: Results from discovery and validation cohorts. Hepatol Commun. 2018;2(7):807-20.

16. Vilar-Gomez E, Chalasani N. Non-invasive assessment of non-alcoholic fatty liver disease: Clinical prediction rules and blood-based biomarkers. Journal of Hepatology. 2018;68(2):305-15.

17. Ekstedt $M$, Hagström $H$, Nasr $P$, Fredrikson $M$, Stål $P$, Kechagias $S$, et al. Fibrosis stage is the strongest predictor for disease-specific mortality in NAFLD after up to 33 years of follow-up. Hepatology. 2015 May;61(5):1547-54.

18. Patel K, Sebastiani G. Limitations of non-invasive tests for assessment of liver fibrosis. JHEP Reports. 2020; 2(2):100067

19. European Association for Study of Liver, Asociacion Latinoamericana para el Estudio del Higado. EASL-ALEH Clinical Practice Guidelines: Noninvasive tests for evaluation of liver disease severity and prognosis. J Hepatol. 2015;63(1):237-64.

20. Xiao G, Zhu S, Xiao X, Yan L, Yang J, Wu G. Comparison of laboratory tests, ultrasound, or magnetic resonance elastography to detect fibrosis in patients with non-alcoholic fatty liver disease: A meta-analysis. Hepatology. 2017;66(5):1486-501.

21. Ampuero J, Pais R, Aller R, Gallego-Durán R, Crespo J, García-Monzón C, et al. Development and Validation of Hepamet Fibrosis Scoring System-A Simple, Non-invasive Test to Identify Patients With Nonalcoholic Fatty Liver Disease With Advanced Fibrosis. Clin Gastroenterol Hepatol. 2020;18(1):216-225.e5. 
22. Ballestri S, Mantovani A, Baldelli E, Lugari S, Maurantonio M, Nascimbeni F, et al. Liver Fibrosis Biomarkers Accurately Exclude Advanced Fibrosis and Are Associated with Higher Cardiovascular Risk Scores in Patients with NAFLD or Viral Chronic Liver Disease. Diagnostics. 2021;11(1):98.

23. Shaheen AAM, Myers RP. Diagnostic accuracy of the aspartate aminotransferase-to-platelet ratio index for the prediction of hepatitis C-related fibrosis: a systematic review. Hepatology. 2007;46(3):912-21.

24. Poynard T, Morra R, Halfon P, Castera L, Ratziu V, Imbert-Bismut F, et al. Meta-analyses of FibroTest diagnostic value in chronic liver disease. BMC Gastroenterol. 2007;7(1):40.

25. Patel PJ, Connoley D, Rhodes F, Srivastava A, Rosenberg W. A review of the clinical utility of the Enhanced Liver Fibrosis test in multiple aetiologies of chronic liver disease. Ann Clin Biochem. 2020;57(1):36-43.

26. Srivastava A, Jong S, Gola A, Gailer R, Morgan S, Sennett K, et al. Costcomparison analysis of FIB-4, ELF and fibroscan in community pathways for non-alcoholic fatty liver disease. BMC Gastroenterol. 2019;19(1):122.

27. Berzigotti A, Tsochatzis E, Boursier J, Castera L, Cazzagon N, Friedrich-Rust $M$, et al. EASL Clinical Practice Guidelines on non-invasive tests for evaluation of liver disease severity and prognosis - 2021 update. J Hepatol. 2021;3:S016882(21):00398-6.

28. McPherson S, Tim H, Dufour JF, Petta S, Romero-Gomez M, Allison M et al. Age as a Confounding Factor for the Accurate Non-Invasive Diagnosis of Advanced NAFLD Fibrosis. Am J Gastroenterol. 2017;112(5):740-51.

29. Mansoor, S., Yerian, L., Kohli, R. et al. The Evaluation of Hepatic Fibrosis Scores in Children with Nonalcoholic Fatty Liver Disease. Dig Dis Sci. 2015;60, 1440-7.
30. Jasmine A Jackson, Juna V Konomi, Michael V Mendoza, Alyssa Krasinskas, Ran Jin, Shelley Caltharp, Marialena Mouzaki MBV. Performance of fibrosis prediction scores in paediatric non-alcoholic fatty liver disease. J Paediatr Child Health. 2018;54(2):172-6.

31. Fedchuk L, Nascimbeni F, Pais R, Charlotte F, Housset C, Ratziu V, et al. Performance and limitations of steatosis biomarkers in patients with nonalcoholic fatty liver disease. Aliment Pharmacol Ther. 2014;40(10):1209-22.

32. Luis C. Bertot, Gary P. Jeffrey, Bastiaan de Boer, Gerry MacQuillan, George Garas, Justin Chin, Yi Huang LAA. Diabetes impacts prediction of cirrhosis and prognosis by non-invasive fibrosis models in non-alcoholic fatty liver disease. Liver Int. 2018;38(10):1793-802.

33. Ooi GJ, Burton PR, Doyle L, Wentworth JM, Bhathal PS, Sikaris K, et al. Modified thresholds for fibrosis risk scores in non-alcoholic fatty liver disease are necessary in the obese. Obes Surg. 2017;27(1):115-25.

34. Joo SK, Kim W, Kim D, Kim JH, Oh S, Lee KL, et al. Steatosis severity affects the diagnostic performances of non-invasive fibrosis tests in non-alcoholic fatty liver disease. Liver Int.2017;38(2):331-41.

35. Angulo P, Hui JM, Marchesini G, Bugianesi E, George J, Farrell GC, et al. The NAFLD Fibrosis Score: A Noninvasive System That Identifies Liver Fibrosis in Patients with NAFLD. Hepatology. 2007;45(4):846-54.

36. Mózes FE, Lee JA, Selvaraj EA, Jayaswal ANA, Trauner M, Boursier J, et al. Diagnostic accuracy of non-invasive tests for advanced fibrosis in patients with NAFLD: an individual patient data meta-analysis. Gut . 2021;0:1-14

37. Higuera-De-La-Tijera F, Córdova-Gallardo J, Buganza-Torio E, BarrancoFragoso B, Torre A, Parraguirre-Martínez S, et al. Hepamet Fibrosis Score in Nonalcoholic Fatty Liver Disease Patients in Mexico: Lower than Expected Positive Predictive Value. Dig Dis Sci. 2021. 